\title{
$\widehat{A}$ Madridge \\ madridge Journal of Otorhinolaryngology \\ interconnecting Scientific World
}

Review Article

Open Access

\section{Evaluation and Treatment of Benign Paroxysmal Positional Vertigo}

\author{
Bonni L Kinne* \\ Department of Physical Therapy, Grand Valley State University, Grand Rapids, MI, USA
}

\section{Article Info}

\author{
*Corresponding author: \\ Bonni L Kinne \\ Department of Physical Therapy \\ Grand Valley State University \\ 301 \\ Grand Rapids, MI 49503 \\ USA \\ Tel. 616-331-5602 \\ E-mail: kinneb@gvsu.edu
}

Received: June 26, 2018

Accepted: July 31, 2018

Published: August 5, 2018

Citation: Kinne BL. Evaluation and Treatment of Benign Paroxysmal Positional Vertigo. Madridge J Otorhinolaryngol. 2018; 3(1): 50-54.

doi: $10.18689 / \mathrm{mjol}-1000110$

Copyright: @ 2018 The Author(s). This work is licensed under a Creative Commons Attribution 4.0 International License, which permits unrestricted use, distribution, and reproduction in any medium, provided the original work is properly cited.

Published by Madridge Publishers

\section{Introduction}

Benign paroxysmal positional vertigo (BPPV), one of the most common peripheral vestibular dysfunctions, occurs when otoconia (calcium carbonate crystals) break loose from the utricle and float into one of the semicircular canals (see Figure 1) [1]. There are three types of BPPV (named according to the affected semicircular canal): [A]. Posterior (or inferior) canal BPPV [2], the most common type [B]. Anterior (or superior) canal BPPV [3], the least common type; and [C]. Horizontal (or lateral) canal BPPV [4]. In addition, there are two subtypes of BPPV (named according to the otoconia's final destination): [A]. Canalithiasis [5], a situation in which the otoconia remain free-floating in the endolymph (a viscous fluid located throughout the inside of each semicircular canal); and $[B]$. Cupulolithiasis [6], a situation in which the otoconia become attached to the cupula (a gelatinous structure located at one end of each semicircular canal). The most common causes of BPPV are the aging process in individuals over the age of 50 [7], traumatic brain injuries in individuals under the age of 50 [8], and inner ear diseases in individuals of all ages [9]. BPPV is generally characterized by vertigo and nystagmus provoked by specific head movements (such as those associated with lying down in bed, rolling over in bed, looking upward, and/or bending over) [1]. The purpose of this review was to outline the most frequently used evaluation and treatment techniques for each type and subtype of BPPV.

Keywords: Evaluation Techniques, Treatment Techniques, Cupulolithiasis, Canalithiasis and Maneuver.

\section{Evaluation Techniques \\ Posterior Canal BPPV}

The gold standard test for posterior canal BPPV is the Dix-Hallpike test (see Figure 2) [2]. Right-sided posterior canal BPPV is diagnosed as follows: [A]. The patient begins in a long-sitting position with the head turned to the right $[B]$. The therapist moves the patient into a supine position with the head slightly extended off the end of the treatment table, and $[\mathrm{C}]$. The patient demonstrates up beating right torsional nystagmus. Left-sided posterior canal BPPV is diagnosed as follows: [A]. The patient begins in a long-sitting position with the head turned to the left [B]. The therapist moves the patient into a supine position with the head slightly extended off the end of the treatment table, and [C]. The patient demonstrates up beating left torsional nystagmus. Canalithiasis of the posterior semicircular canal is characterized by a long latency in the onset of the nystagmus, a short duration of the evoked nystagmus, and fatigability of the nystagmus upon repeated testing. Although cupulolithiasis of the posterior semicircular canal is also characterized by fatigability of the nystagmus upon repeated testing, the latency in the onset of the nystagmus is shorter and the duration of the evoked nystagmus is longer. 


\section{Anterior Canal BPPV}

The gold standard test for anterior canal BPPV is the head hanging test (see Figure 3) [10]. Right-sided anterior canal BPPV is diagnosed as follows: $[A]$. The patient begins in a long-sitting position with the head in neutral $[B]$. The therapist moves the patient into a supine position with the head completely extended off the end of the treatment table, and $[\mathrm{C}]$. The patient demonstrates down beating right torsional nystagmus. Leftsided anterior canal BPPV is diagnosed as follows: [A]. The patient begins in a long-sitting position with the head in neutral [B]. The therapist moves the patient into a supine position with the head completely extended off the end of the treatment table, and [C]. The patient demonstrates down beating left torsional nystagmus. Canalithiasis of the anterior semicircular canal is characterized by a long latency in the onset of the nystagmus, a short duration of the evoked nystagmus, and fatigability of the nystagmus upon repeated testing. Although cupulolithiasis of the anterior semicircular canal is also characterized by fatigability of the nystagmus upon repeated testing, the latency in the onset of the nystagmus is shorter and the duration of the evoked nystagmus is longer.

\section{Horizontal Canal BPPV}

The gold standard test for diagnosing horizontal canal BPPV is the head roll test (see Figure 4) [11]. A geotropic presentation occurs when the otoconia are free-floating in the posterior arm of the horizontal semicircular canal (canalithiasis), and this presentation is diagnosed as follows: $[A]$. The patient begins in a supine position with the head completely flexed [B]. The therapist turns the patient's head to the right $[C]$. The patient demonstrates right-beating nystagmus [D]. The patient returns to a supine position with the head completely flexed [E]. The therapist turns the patient's head to the left, and [F]. The patient demonstrates left-beating nystagmus. An ageotropic presentation occurs when the otoconia are free-floating in the anterior arm of the horizontal semicircular canal (canalithiasis) or are attached to the cupula in the horizontal semicircular canal (cupulolithiasis), and this presentation is diagnosed as follows: $[A]$. The patient begins in a supine position with the head completely flexed [B]. The therapist turns the patient's head to the right $[C]$. The patient demonstrates left-beating nystagmus [D]. The patient returns to a supine position with the head completely flexed [E]. The therapist turns the patient's head to the left, and [F]. The patient demonstrates right-beating nystagmus. In both cases, the affected ear is the one toward which the greatest nystagmus occurs.

\section{Treatment Techniques Posterior Canal BPPV}

Although canalithiasis of the posterior semicircular canal is most commonly treated with the Epley maneuver [12], this intervention has been modified several times since its inception in 1992. One such modification is the Parnes maneuver (see Figure 5) [13]. For right-sided canalithiasis of the posterior semicircular canal, the Parnes maneuver is performed as follows: $[A]$. The patient begins in a long-sitting position with the head turned to the right $[\mathrm{B}]$. The therapist moves the patient into a supine position with the head slightly extended off the end of the treatment table [C]. The patient remains in this position for 2 to 3 minutes after the evoked nystagmus has resolved [D]. The therapist moves the patient into a left side-lying position with the head turned to the left, and $[\mathrm{E}]$. The patient remains in this position for 1 to 2 minutes after the evoked nystagmus has resolved. For left-sided canalithiasis of the posterior semicircular canal, the Parnes maneuver is performed as follows: $[A]$. The patient begins in a long-sitting position with the head turned to the left $[B]$. The therapist moves the patient into a supine position with the head slightly extended off the end of the treatment table [C]. The patient remains in this position for 2 to 3 minutes after the evoked nystagmus has resolved [D]. The therapist moves the patient into a right side-lying position with the head turned to the right, and [E]. The patient remains in this position for 1 to 2 minutes after the evoked nystagmus has resolved. In their initial study, Parnes and Price-Jones [13] reported an overall success rate of $88 \%$.

Cupulolithiasis of the posterior semicircular canal is most commonly treated with the Semont maneuver (see Figure 6) [14]. For right-sided cupulolithiasis of the posterior semicircular canal, the Semont maneuver is performed as follows: $[A]$. The patient begins in a sitting position with the head turned to the left $[B]$. The therapist moves the patient into a right-sidelying position [C]. The patient remains in this position for 2 to 3 minutes after the evoked nystagmus has resolved [D]. The therapist moves the patient up into a sitting position and down into a left side-lying position in one continuous motion, and $[\mathrm{E}]$. The patient remains in this position for 5 minutes after the evoked nystagmus has resolved. For left-sided cupulolithiasis of the posterior semicircular canal, the Semont maneuver is performed as follows: $[A]$. The patient begins in a sitting position with the head turned to the right $[B]$ The therapist moves the patient into a left side-lying position $[C]$. The patient remains in this position for 2 to 3 minutes after the evoked nystagmus has resolved [D]. The therapist moves the patient up into a sitting position and down into a right side-lying position in one continuous motion, and [E]. The patient remains in this position for 5 minutes after the evoked nystagmus has resolved. In their initial study, Semont et al [14] reported a success rate of $84 \%$ after one treatment session and $93 \%$ after two treatment sessions.

\section{Anterior Canal BPPV}

The Parnes maneuver may be effective for canalithiasis of the anterior semicircular canal, and the Semont maneuver may be effective for cupulolithiasis of the anterior semicircular canal. However, a recent systematic review described interventions specifically designed for the treatment of anterior canal BPPV [15]. Two of the interventions described in this systematic review are capable of eliminating a patient's vertigo even if the affected ear is difficult to identify.

The first of these two interventions was developed by Yacovino et al [16] in 2009 (see Figure 7). This maneuver is performed as follows: $[A]$. The patient begins in a long-sitting position with the head in neutral $[B]$. The therapist moves the patient into a supine position with the head extended at least 30 degrees off the end of the treatment table [C]. The patient remains in this position for 30 seconds [D]. The therapist 
completely flexes the patient's head [E]. The patient remains in this position for 30 seconds, and [F]. The therapist moves the patient into a long-sitting position with the head in neutral. In their initial study, Yacovino et al [16] reported a success rate of $85 \%$ after one treatment session and $100 \%$ after two treatment sessions.

The second of these two interventions was developed by Casani et al [17] in 2011 (see Figure 7). This maneuver is performed as follows: $[A]$. The patient begins in a long-sitting position with the head in neutral $[B]$. The therapist moves the patient into a supine position with the head completely extended off the end of the treatment table [C]. The patient remains in this position for 3 minutes [D]. The therapist completely flexes the patient's head [E]. The patient remains in this position for 3 minutes [F]. The therapist moves the patient into a long-sitting position with the head in neutral, and [G]. Steps A-F are repeated. In their initial study, Casani et al [17] reported a success rate of $44 \%$ after one treatment session and $83 \%$ after two treatment sessions.

\section{Horizontal Canal BPPV}

The effective treatment of horizontal canal BPPV is dependent upon the proper identification of both the presentation (geotropic vs. ageotropic) and the affected ear (right vs. left). A recent systematic review described interventions designed for the treatment of the two horizontal canal BPPV presentations [18]. If the affected ear is difficult to identify, accessory diagnostic procedures may need to be used in conjunction with the head roll test [19]. Although many of the original horizontal canal BPPV maneuvers involved a 180-degree [20], 270-degree [21], or 360-degree [22] roll, side-lying interventions have been more recently described for each type of presentation.

The most commonly used side-lying intervention for the geotropic presentation of horizontal canal BPPV was described by Appiani et al [23] in 2001 (see Figure 8). For right-sided geotropic horizontal canal BPPV, this maneuver is performed as follows: $[A]$. The patient begins in a sitting position with the head in neutral $[B]$. The therapist moves the patient into a left side-lying position $[\mathrm{C}]$. The patient remains in this position for 2 minutes [D]. The therapist turns the patient's head to the left, and [E]. The patient remains in this position for 2 minutes. For left-sided geotropic horizontal canal BPPV, this maneuver is performed as follows: $[A]$. The patient begins in a sitting position with the head in neutral $[B]$. The therapist moves the patient into a right side-lying position $[C]$. The patient remains in this position for 2 minutes [D]. The therapist turns the patient's head to the right, and [E]. The patient remains in this position for 2 minutes. In their initial study, Appiani et al [23] reported a success rate of $78 \%$ after one treatment session and $100 \%$ after two treatment sessions.

The most commonly used side-lying intervention for the ageotropic presentation of horizontal canal BPPV was described by Appiani et al [24] in 2005 (see Figure 9). For right-sided ageotropic horizontal canal BPPV, this maneuver is performed as follows: $[\mathrm{A}]$. The patient begins in a sitting position with the head in neutral $[B]$. The therapist moves the patient into a right side-lying position $[C]$. The patient remains in this position for 2 minutes [D]. The therapist turns the patient's head to the left, and [E]. The patient remains in this position for 2 minutes. For left-sided ageotropic horizontal canal BPPV, this maneuver is performed as follows: $[A]$. The patient begins in a sitting position with the head in neutral [B]. The therapist moves the patient into a left side-lying position [C]. The patient remains in this position for 2 minutes [D]. The therapist turns the patient's head to the right, and [E]. The patient remains in this position for 2 minutes. In their initial study, Appiani et al [24] reported a success rate of $100 \%$ after one treatment session.

\section{Final Thoughts}

In general, clinician-directed maneuvers are very successful for eliminating the vertigo and nystagmus associated with BPPV. However, the persistence of BPPV and the recurrence of BPPV are possible.

In terms of BPPV persistence, Dorigueto et al [25] found that $4 \%$ of participants continued to experience vertigo and nystagmus despite undergoing repeated maneuvers over the course of one year. In cases of persistent BPPV, a comprehensive re-evaluation should be conducted [26]. During this reevaluation, the clinician should determine whether the patient has a type of BPPV that may be successfully treated with a different maneuver, another vestibular dysfunction that may be successfully treated with a vestibular rehabilitation home program, or a central nervous system disorder that may need to be addressed by another healthcare professional. For persistent cases of BPPV that do not respond to any type of maneuver, a vestibular nerve section or a semicircular canal plugging surgical procedure may be indicated.

In terms of BPPV recurrence, Dorigueto et al [25] found that $26 \%$ of participants experienced a new onset of vertigo and nystagmus within one year of undergoing a successful maneuver. Although "medical research has not found any way to stop BPPV from coming back, it can be treated with a high rate of success [through the administration of another maneuver]" [26], [pS36].

Figure 1: The peripheral vestibular system depicting the utricle and the semicircular canals (image designed by Tess Tobolic).

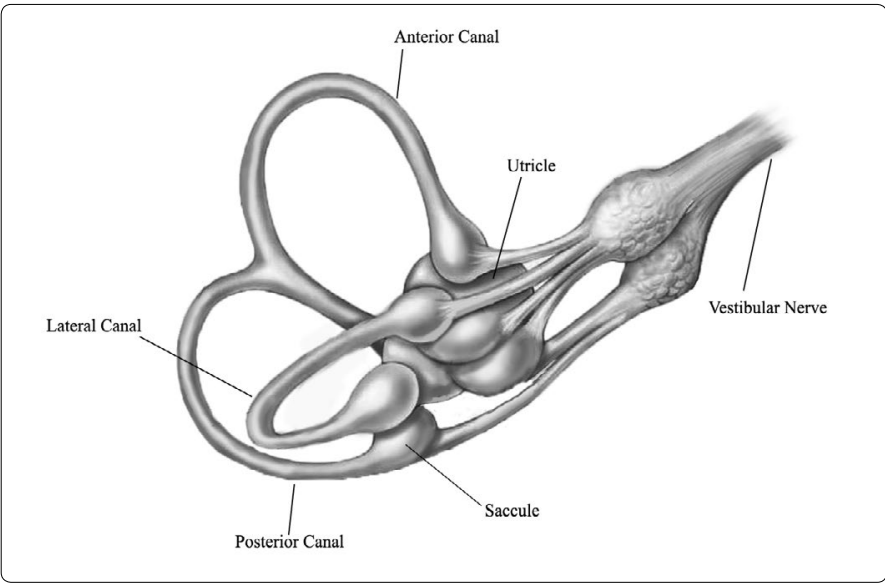


Figure 2: The Dix-Hallpike Test.

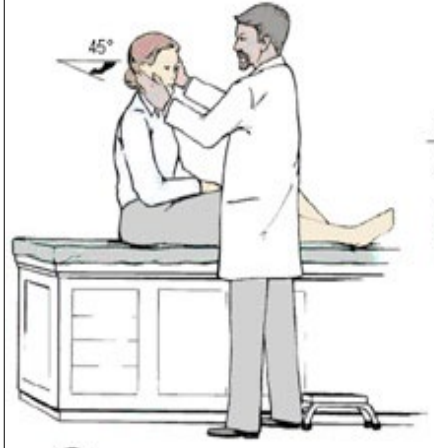

(A)

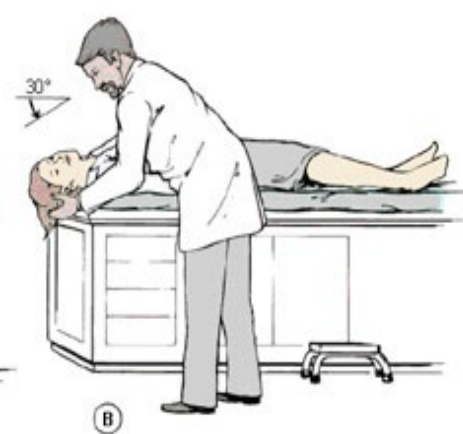

(B)

Figure 3: The Head Hanging Test.

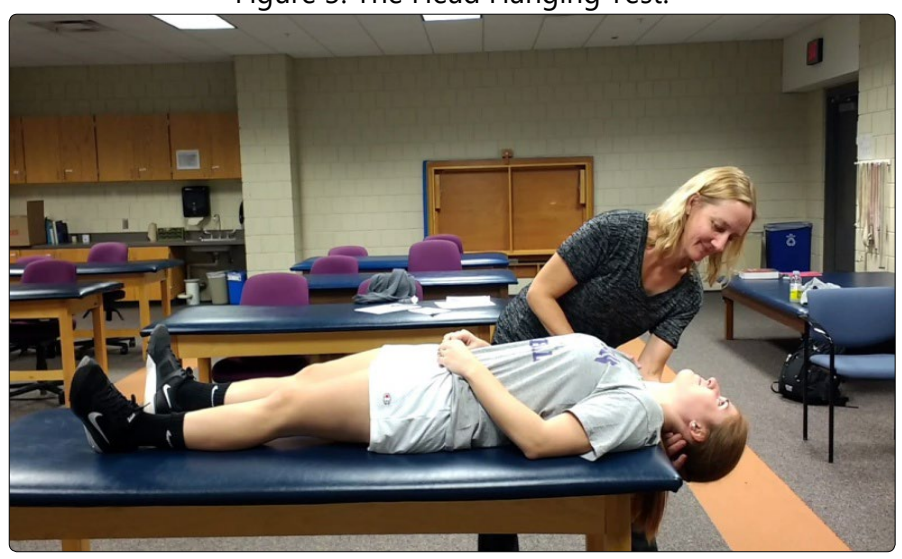

Figure 4: The Head Roll Test.

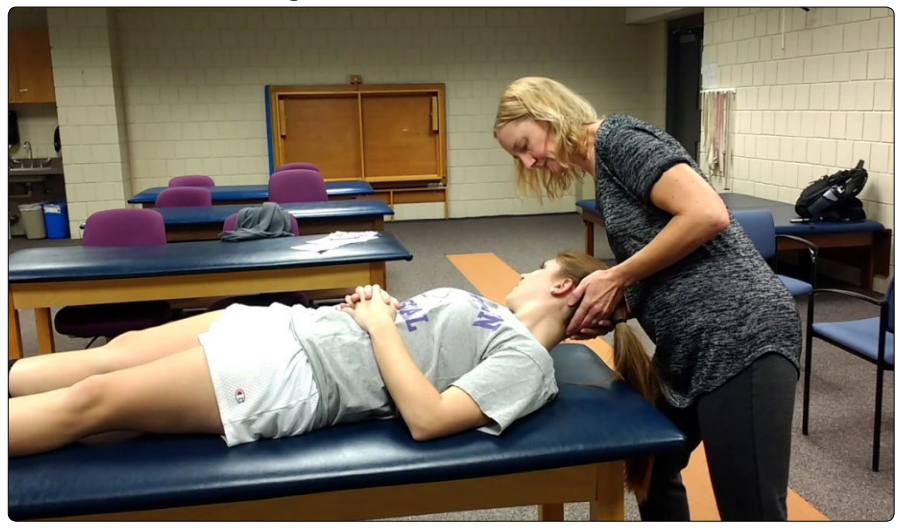

Figure 5: The Parnes Maneuver.

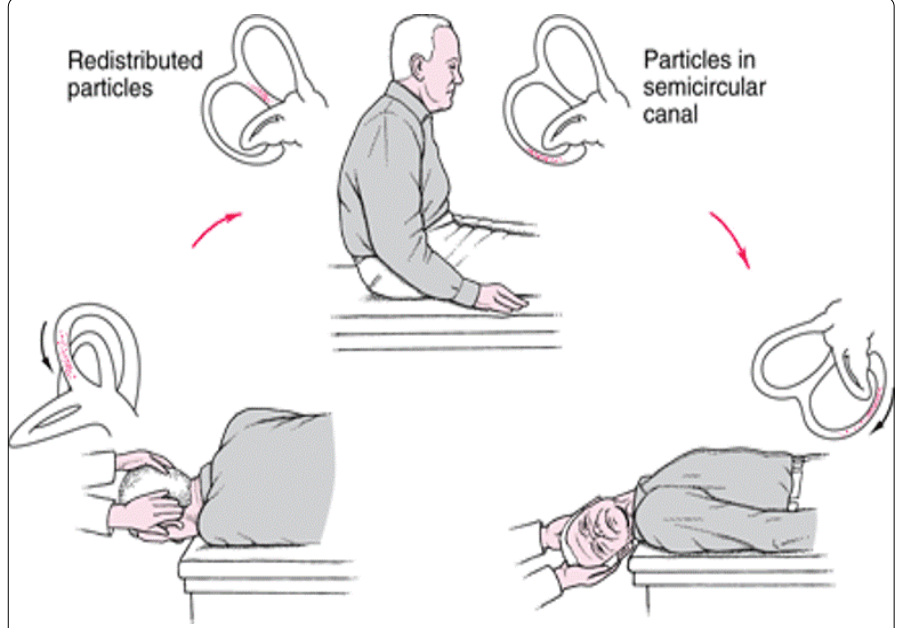

Figure 6: The Semont Maneuver.

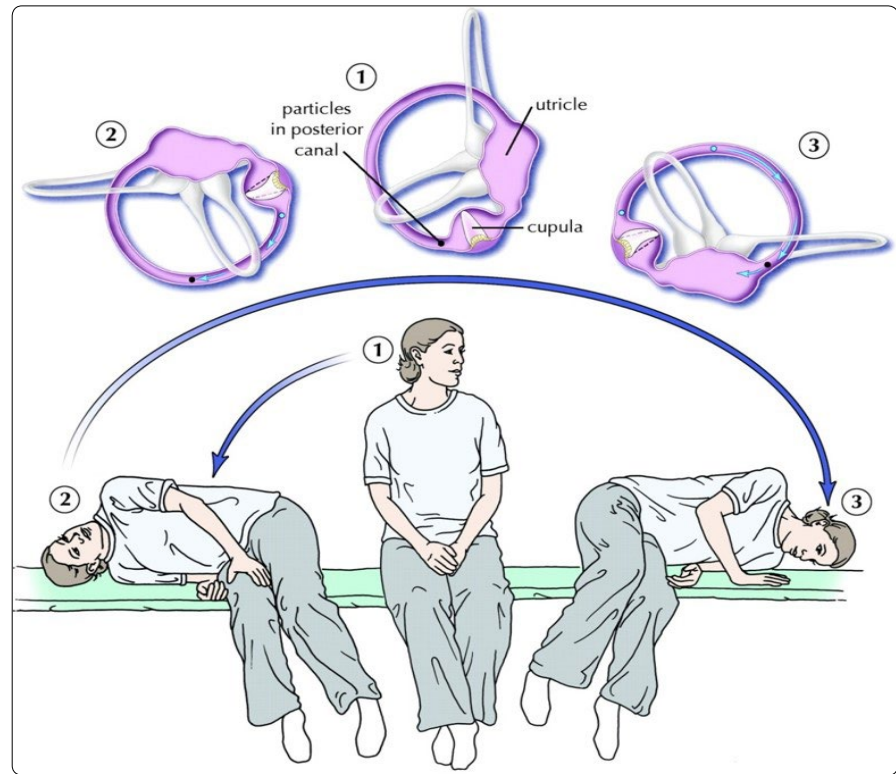

Figure 7: Specific Anterior Canal BPPV Maneuver.

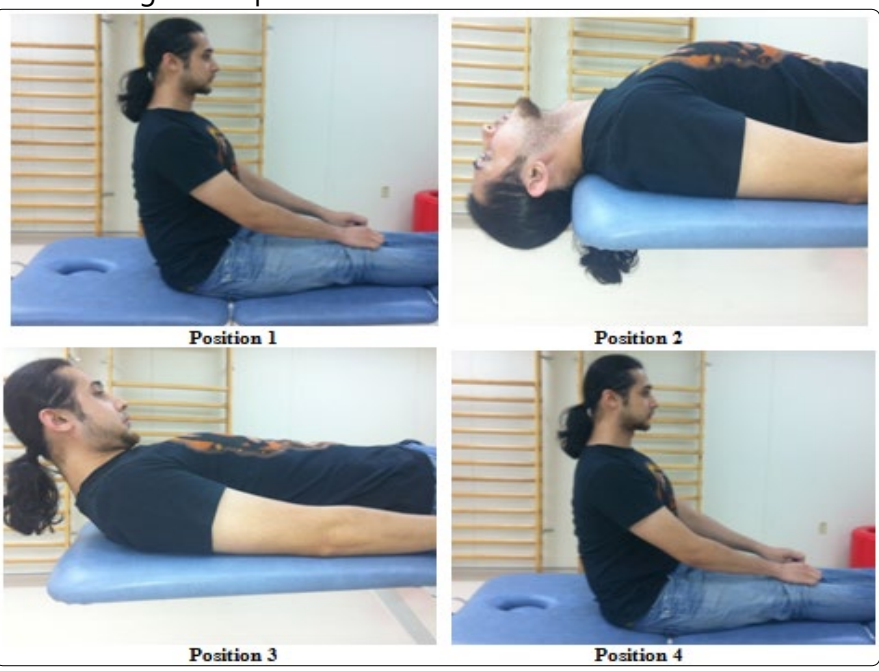

Figure 8: Geotropic Horizontal Canal BPPV Maneuver.
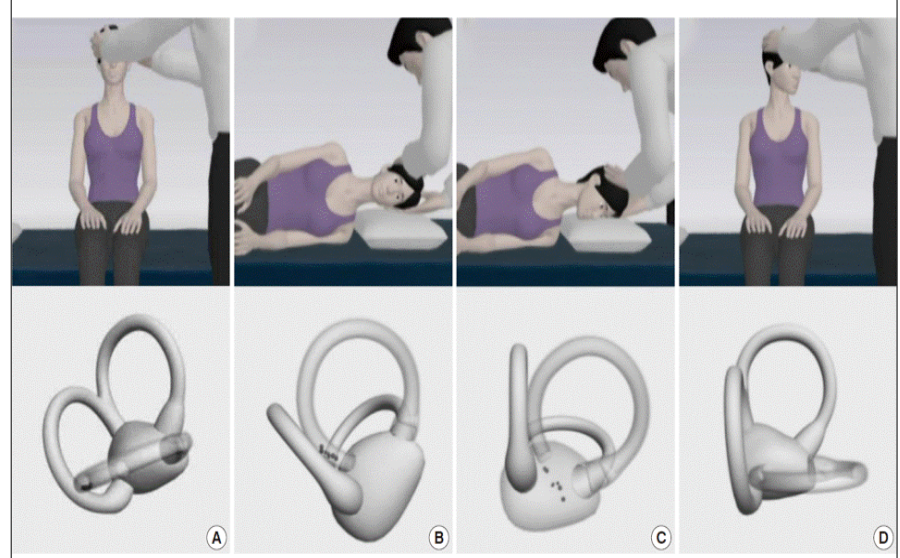
Figure 9: Ageotropic Horizontal Canal BPPV Maneuver.

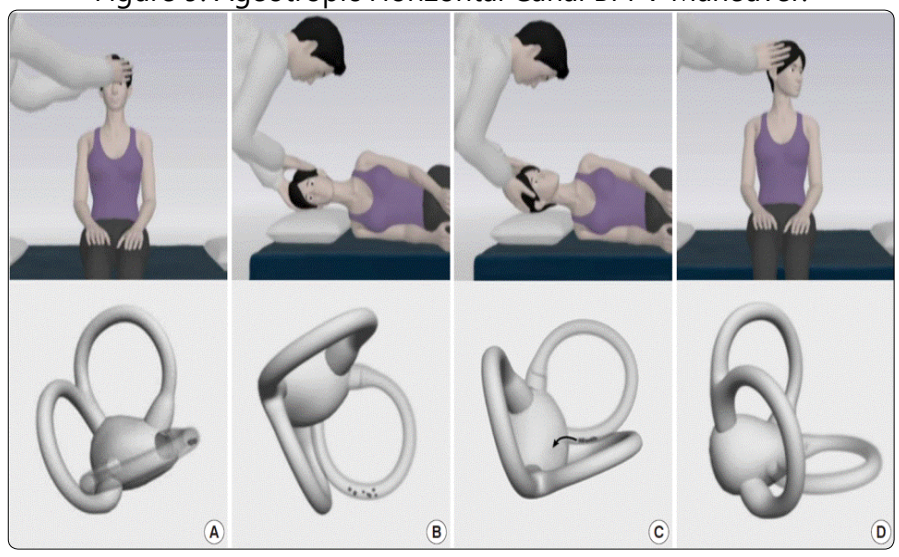

\section{References}

1. Kim JS, Zee DS. Benign paroxysmal positional vertigo. N Engl J Med. 2014; 370: 1138-47. doi: 10.1056/NEJMcp1309481

2. Dix MR, Hallpike CS. The pathology, symptomatology and diagnosis of certain common disorders of the vestibular system. Proc R Soc Med. 1952; 45: 341-54.

3. Katsarkas A. Nystagmus of paroxysmal positional vertigo: some new insights. AnnOtolRhinolLaryngol.1987;96:305-308.doi:10.1177/000348948709600313

4. Mc Clure JA. Horizontal canal BPV. J Otolaryngol. 1985; 14: 30-35.

5. Hall SF, Ruby RRF, McClure JA. The mechanics of benign paroxysmal vertigo. J Otolaryngol. 1979; 8: 151-158.

6. Schuknecht HF. Cupulolithiasis. Arch Otolaryngol. 1969; 90: 765-778. doi: 10.1001/archotol.1969.00770030767020

7. Faralli M, Ricci G, Molini E, Bressi T, Simoncelli C, et al. Paroxysmal positional vertigo: the role of age as a prognostic factor. Acta Otorhinolaryngol Ital. 2006; 26: 25-31

8. Ahn SK, Jeon SY, Kim JP, park JJ, Hur DG, et al. Clinical characteristics and treatment of benign paroxysmal positional vertigo after traumatic brain injury. J Trauma. 2011; 70: 442-446. doi: 10.1097/TA.0b013e3181d0c3d9.

9. Lee $\mathrm{NH}$, Ban JH, Lee KC, Kim SM. Benign paroxysmal positional vertigo secondary to inner ear disease. Otolaryngol Head Neck Surg. 2010; 143: 413-417. doi:10.1016/j.otohns.2010.06.905.

10. Bertholon P, Bronstein AM, Davies RA, Rudge P, Thilo KV. Positional down beating nystagmus in 50 patients: cerebellar disorders and possible anterior semicircular canalithiasis. J Neurol Neurosurg Psychiatry. 2002; 72: 366-372.
11. Pagnini $P$, Nuti $D$, Vannucchi $P$. Benign paroxysmal vertigo of the horizontal canal. Orl J Otorhinolaryngol Relat Spec. 1989; 51: 161-170.

12. Epley JM. The canalith repositioning procedure: for treatment of benign paroxysmal positional vertigo. Otolaryngol Head Neck Surg. 1992; 107: 399-404. doi: 10.1177/019459989210700310

13. Parnes $L S$, Price-Jones R. Particle repositioning maneuver for benign paroxysmal positional vertigo. Ann Otol Rhinol Laryngol. 1993; 102: 325331. doi: $10.1177 / 000348949310200501$

14. Semont $A$, Freyss $G$, Vitte $E$. Curing the BPPV with a liberatory maneuver. Adv Otorhinolaryngol. 1988; 42: 290-293.

15. Kinne $\mathrm{BL}$, Crouch NA, Strace $\mathrm{CL}$. Anterior canal benign paroxysmal positional vertigo treatment techniques. Phys Ther Rev. 2014; 19: 79-85. doi: 10.1179/1743288X13Y.0000000112

16. Yacovino DA, Hain TC, Gualtieri F. New therapeutic maneuver for anterior canal benign paroxysmal positional vertigo. J Neurol. 2009; 256: 18511855. doi: 10.1007/s00415-009-5208-1

17. Casani AP, Cerchiai N, Dallan I, Sellari-Francescini S. Anterior canal lithiasis: diagnosis and treatment. Otolaryngol Head Neck Surg. 2011; 144: 412418. doi: $10.1177 / 0194599810393879$

18. Kinne $B L$, Strace $C L$, Crouch NA. The effectiveness of current interventions in the management of lateral canal benign paroxysmal positional vertigo. Phys Ther Rev. 2012; 17: 292-300. doi: 10.1179/1743288X12Y.0000000028

19. Kinne BL, Baker BJ, Harro CC. Identification of the affected ear in lateral canal benign paroxysmal positional vertigo. Phys Ther Rev. 2014; 19: 293 301. doi: 10.1179/1743288X14Y.0000000149

20. Baloh RW, Jacobson K, Honrubia V. Horizontal semicircular canal variant of benign positional vertigo. Neurology. 1993; 43: 2542-2549.

21. Lempert T. Horizontal benign positional vertigo. Neurology. 1994; 44: 2213-2214.

22. Baloh RW. Horizontal benign positional vertigo. Neurology. 1994; 44: 2214.

23. Appiani GC, Catania G, Gagliardi M. A liberatory maneuver for the treatment of horizontal canal paroxysmal positional vertigo. Otol Neurotol. 2001; 22: 66-69. doi: 10.1097/00129492-200101000-00013.

24. Appiani GC, Catania G, Gagliardi M, Cuiuli G. Repositioning maneuver for the treatment of the apogeotropic variant of horizontal canal benign paroxysmal positional vertigo. Otol Neurotol. 2005; 26: 257-260.

25. Dorigueto RS, Mazzetti KR, Gabilan YPL, Gananca FF. Benign paroxysmal positional vertigo recurrence and persistence. Braz J Otorhinolaryngol. 2009; 75: 565-572. doi: 10.1590/S1808-86942009000400016

26. Bhattacharyya N, Gubbels SP, Schwartz SR, Edlow JA, El-Kashlan H, et al. Clinical practice guideline: benign paroxysmal positional vertigo (update). Otolaryngol Head Neck Surg. 2017; 156: S1-S47. doi: 10.1177/0194599816689667. 